\title{
Isotretinoin Meets COVID-19: Revisiting a Fragmented Paradigm
}

\section{Lorraine L. Rosamilia, MD \\ We cannot solve our problems with the same thinking we used when we created them.}

\section{Albert Einstein}

$\mu$ midst the myriad of disruptions and corollary solutions budding from the ongoing global COVID-19 pandemic, management of acne with isotretinoin underwent a makeover. Firstly, as with any pharmaceutical prescribed in the last 1 to 2 years, patients asked the compelling question, "Will this prescription put me at higher risk for COVID-19?", resulting in a complex set of answers from both clinical and basic science perspectives. Further, the practical use of telemedicine for clinical visits and pregnancy test reporting altered the shape of isotretinoin physician-patient communication and follow-up. Finally, the combination of these circumstances spurred us to revisit common quandaries in prescribing this drug: Can we trust what patients tell us when they are taking isotretinoin? Do we need to monitor laboratory values and follow patients on isotretinoin as closely and as frequently as we have in the past? Does the Risk Evaluation and Mitigation Strategy (REMS) program of iPLEDGE hold true utility?

\section{Impact of COVID-19 on Isotretinoin Use}

Isotretinoin may have varying influence on the ease of host entry and virulence of COVID-19. Because the majority of patients experience some degree of mucous membrane desiccation on isotretinoin, it originally was postulated that disruption of the nasal mucosa, thereby uncovering the basal epithelial layer where angiotensin-converting enzyme 2 (ACE2) receptors are expressed, could increase the risk for viral invasion, as ACE2 is the host receptor for COVID-19 entry. ${ }^{1,2}$ On the other hand, a study of 672 medications and their effect on regulation of ACE2 levels stratified isotretinoin in the highest category of ACE2 downregulators, therefore theoretically preventing cellular entry and replication of the virus. ${ }^{3}$ In conferring with many of my colleagues and reviewing available literature, I found that these data did not summarily deter providers from initiating or continuing isotretinoin during the pandemic, and research is ongoing to particularly earmark isotretinoin as a possible COVID-19 therapy option. ${ }^{4,5}$ Despite this, and despite the lower risk for COVID-19 in the customary isotretinoin adolescent and young adult age range, an Italian study reported that $14.7 \%$ of patients (5/34) prematurely interrupted isotretinoin therapy during lockdown because of fear of COVID-19 infection. ${ }^{6}$ Data also suggest that college towns (akin to where I practice, rife with isotretinoin-eligible patients) reflected higher COVID-19 infection and death rates, likely due to dense cohabitation and intermittent migration of students and staff to and from campuses and within their communities. ${ }^{7}$ Approximately 30\% of my patients on isotretinoin in the last 18 months reported having COVID-19 at some point during the pandemic, though no data exist to guide us on whether isotretinoin should be discontinued in this scenario; my patients typically continued the drug unless their primary health care team discouraged it, and in those cases, all of them resumed it after COVID-19 symptomatology resolved.

Last spring, the US Department of Health and Human Services and the US Food and Drug Administration announced that health care professionals who prescribe and/or dispense drugs subject to REMS with laboratory testing or imaging requirements should consider whether there are compelling reasons not to complete the required testing/ imaging during the current public health emergency and use their best medical judgment in weighing the benefits and risks of continuing treatment in the absence of these tests. It also was stressed that prescribers should effectively communicate with their patients regarding these benefits, risks, and altered protocols. ${ }^{8}$ Further, the iPLEDGE program concurred that telemedicine was an acceptable visit type for both initiating and maintaining isotretinoin, and home pregnancy tests were valid for females of childbearing potential if an accurate testing date and results were communicated by patients to the prescriber in the required reporting windows. ${ }^{9}$ This allowed dermatologists to foster what was one of our most important roles as outpatient clinicians during the pandemic: to maintain normalcy, continuity, and support for as many patients as possible.

From the Department of Dermatology, Geisinger Scenery Park, State College, Pennsylvania.

The author reports no conflict of interest.

Correspondence: Lorraine L. Rosamilia, MD, 200 Scenery Dr, 56-02, State College, PA 16801 (Ilrosamilia@geisinger.edu).

doi:10.12788/cutis.0299 


\section{Isotretinoin and Telemedicine}

During the pandemic, continuation of isotretinoin therapy proved easier than initiating it, given that patients could access and maintain a clear connection to the online visit platform, display understanding of the REMS mandates (along with a guardian present for a minor), perform a home pregnancy test and report the result followed by the quiz (for females), and collect the prescription in the allotted window. For new patients, the absence of a detailed in-person examination and rapport with the patient (and guardians when applicable) as well as misalignment of the date of iPLEDGE registration with the timing of the pregnancy test results and prescribing window were more onerous using digital or mailed versions of consent forms and photodocumentation of urine pregnancy test results. This tangle of requirements perpetuated missed prescribing windows, increased patient portal and phone messages, resulted in more time on the phone with the iPLEDGE help desk, and intensified angst for clinical staff.

These telemedicine visits also required validation of the patient's geographic location to verify the billability of the visit and whether the patient was in a secure location to have a US Health Insurance Portability and Accountability Act-compliant conversation as well as the abstract notion that the timing and result of the pregnancy tests for females reflected a true nonpregnant state. ${ }^{10,11}$ Verification of the pregnancy tests in these situations was approached by either the patient reporting the outcome verbally or displaying the pregnancy test kit result in a video or photograph form for the medical record, all of which leave room for error, doubt, and lower sensitivity than laboratory-based collection. That being said, the increased implementation of telemedicine visits during the pandemic sustained patient access, decreased cost with less laboratory testing and reduced time away from work or school, and resulted in high patient satisfaction with their care. ${ }^{12}$ Additionally, it allowed providers to continue to more comfortably inch away from frequent in-person serologic cholesterol and hepatic testing during therapy based on mounting data that it is not indicated. ${ }^{13}$

Accordingly, the complicated concepts of trust, practicality, and sustainability for the safe and effective management of isotretinoin patients re-emerged. For example, prior to COVID-19, we trusted patients who said they were regularly taking their oral contraceptives or were truly practicing abstinence as a form of contraception. During the pandemic, we then added a layer of trust with home pregnancy test reporting. If the patient or guardian signed the isotretinoin consent form and understood the risks of the medication, ideally the physician-patient relationship fostered the optimal goals of honest conversation, adherence to the drug, safety, and clear skin. However, there is yet another trust assay: iPLEDGE, in turn, trusts that we are reporting patient data accurately, provoking us to reiterate questions we asked ourselves before the pandemic. Is the extra provider and staff clerical work and validation necessary, compounded by prior data that iPLEDGE's capacity to limit pregnancyrelated morbidity with isotretinoin has been called into question in the last decade ${ }^{14}$ Do males need to be followed every month? Is laboratory monitoring still necessary for all isotretinoin candidates? Will post-COVID-19 data show that during various versions of the lockdown, an increased number of isotretinoin patients developed unmonitored morbidity, including transaminitis, hypertriglyceridemia, and an increase in pregnancies? How long will telemedicine visits for isotretinoin be reimbursable beyond the pandemic? Are there other models to enhance and improve isotretinoin teledermatology and compliance? ${ }^{15}$

\section{Final Thoughts}

Dermatologists' experience managing high volumes of isotretinoin patients paired with the creativity to maintain meaningful (and truthful) patient connections and decrease administrative burden lie front and center in 2021. Because the COVID-19 pandemic remains ambient with a dearth of data to guide us, I pose the questions above as points for commiseration and catapults for future study, discussion, collaboration, and innovation. Perhaps the neo-COVID-19 world provided us with the spark we needed to metaphorically clean up the dusty isotretinoin tenets that have frayed our time and patience so we can maintain excellent care for this worthy population.

\section{REFERENCES}

1. Hamming I, Timens W, Bulthuis MLC, et al. Tissue disruption of ACE2 protein, the functional receptor for SARS coronavirus. a first step in understanding SARS pathogenesis. J Pathol. 2004;203:631-637.

2. British Association of Dermatologists. COVID-19-isotretinoin guidance. Published March 26, 2020. Accessed June 21, 2021. https://www.bad.org.uk/shared/get-file.ashx?itemtype=document\&id=6661

3. Sinha S, Cheng K, Schäffer AA, et al. In vitro and in vivo identification of clinically approved drugs that modify ACE2 expression. Mol Syst Biol. 2020;16:E9628

4. Öğüt ND, Kutlu Ö, Erbağcr E. Oral isotretinoin treatment in patients with acne vulgaris during the COVID-19 pandemic: a retrospective cohort study in a tertiary care hospital [published online April 22, 2021]. J Cosmet Dermatol. doi:10.1111/jocd.14168

5. Isotretinoin in treatment of COVID-19. National Library of Medicine website. ClinicalTrials.gov identifier: NCT04361422. Updated September 23, 2020. Accessed June 21, 2021. https://clinicaltrials.gov /ct2/show/NCT04361422

6. Donnarumma M, Nocerino $M$, Lauro W, et al. Isotretinoin in acne treatment during the coronavirus disease 2019 (COVID-19): a retrospective analysis of adherence to therapy and side effects [published online December 22, 2020]. Dermatol Ther. 2021;34:E14677.

7. Ivory D, Gebeloff R, Mervosh S. Young people have less COVID-19 risk, but in college towns, deaths rose fast. The New York Times. December 12, 2020. Accessed June 21, 2021. https://www.nytimes .com/2020/12/12/us/covid-colleges-nursing-homes.html

8. US Food and Drug Administration. Coronavirus (COVID-19) update: FDA provides update on patient access to certain REMS drugs during COVID-19 public health emergency. Published March 22, 2020. Accessed June 21, 2021. https://www.fda.gov/news-events /press-announcements/coronavirus-covid-19-update-fda-provides -update-patient-access-certain-rems-drugs-during-covid-19

9. Haelle T. iPledge allows at-home pregnancy tests during pandemic. Dermatology News. Published April 3, 2020. Accessed June 28, 2021. https://www.mdedge.com/dermatology/article/220186/acne/ipledge -allows-home-pregnancy-tests-during-pandemic

10. Bressler MY, Siegel DM, Markowitz O. Virtual dermatology: a COVID-19 update. Cutis. 2020;105:163-164; E2.

CONTINUED ON PAGE 12 


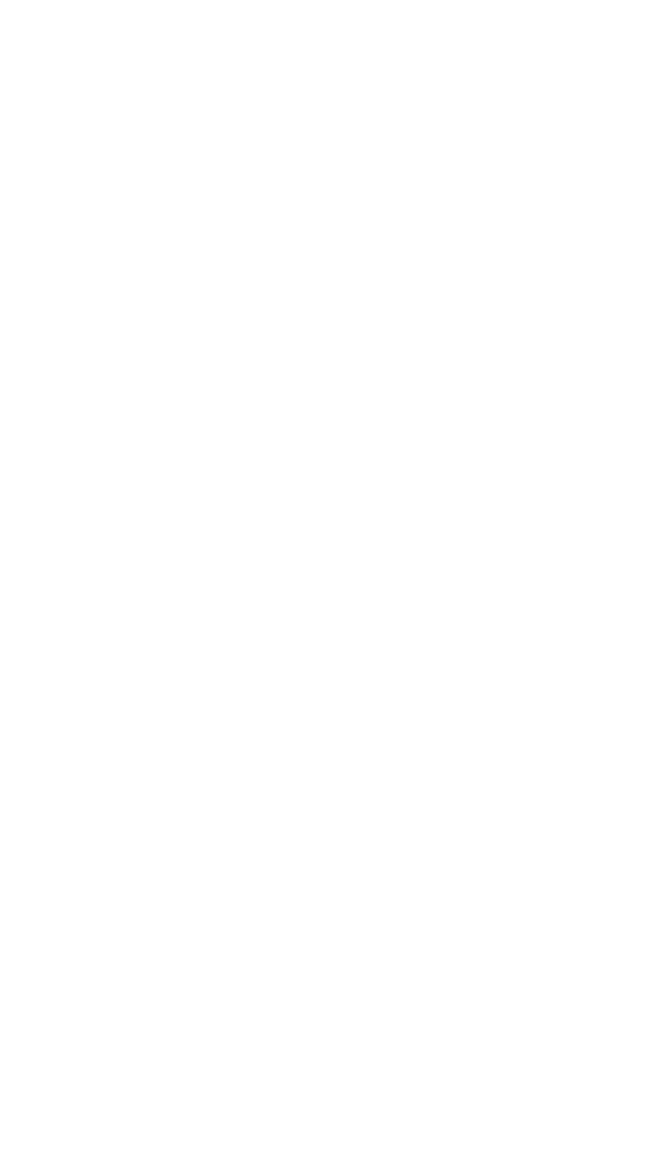

\section{EDITORIAL}

CONTINUED FROM PAGE 9

11. Telemedicine key issues and policy. Federation of State Medical Boards website. Accessed June 28, 2021. https://www.fsmb.org /advocacy/telemedicine

12. Ruggiero A, Megna M, Annunziata MC, et al. Teledermatology for acne during COVID-19: high patients' satisfaction in spite of the emergency. J Eur Acad Dermatol Venereol. 2020;34:E662-E663.

13. Barbieri JS, Shin DB, Wang $S$, et al. The clinical utility of laboratory monitoring during isotretinoin therapy for acne and changes to monitoring practices over time. J Am Acad Dermatol. 2020;82:72-79.

14. Tkachenko E, Singer S, Sharma $\mathrm{P}$, et al. US Food and Drug Administration reports of pregnancy and pregnancy -related adverse events associated with isotretinoin. JAMA Dermatol. 2019;155:1175-1179.

15. Das $\mathrm{S}$, et al. Asynchronous telemedicine for isotretinoin management: a direct care pilot [published online January 21, 2021]. J Am Acad Dermatol. doi:10.1016/j.jaad.2021.01.039 